\title{
Effects of supervised training compared to unsupervised training on physical activity, muscular endurance, and cardiovascular parameters
}

\begin{abstract}
Evidence has suggested that among untrained individuals, supervised exercise interventions may elicit greater health benefits and long-term exercise adherence compared to unsupervised exercise interventions.
\end{abstract}

Purpose: This study focused on determining the effectiveness of a five-month supervised exercise intervention compared to a five-week unsupervised duration at a university worksite for faculty and staff members on physical activity behavior, muscular endurance, and cardiovascular health.

Methods: An exercise intervention met three times per week for five months to undergo 60 minutes of structured, group exercise led by fitness professionals. This was followed by 5 weeks of independent exercise. Data collected included physical activity behavior via a validated accelerometer, muscular endurance, and cardiovascular health.

Results: Repeated measures ANOVA at three time periods were conducted and post-hoc paired samples t-tests to determine where the main effect occurred. The data indicated that participation in a five-month supervised exercise program resulted in improvements of physical activity behavior $(\mathrm{p}=0.003)$ and increased muscular endurance via push-ups $(\mathrm{p}=0.002)$ and curl-ups $(\mathrm{p}<0.001)$. A five-week unsupervised program resulted in decreased physical activity behavior, decreased muscular endurance back to baseline levels, and increased resting heart rate $(\mathrm{p}=0.005)$.

Conclusion: This study suggests that supervised exercise programs for previously sedentary individuals are effective in improving overall fitness. While a period of unsupervised exercise may result in less physical activity and decrease in muscular endurance and cardiovascular health.
Volume 5 Issue 4 - 2016

\author{
Curtis Fennell, Kylene Peroutky, Ellen L \\ Glickman \\ Exercise Physiology, Kent State University, USA
}

\begin{abstract}
Correspondence: Curtis Fennell, Exercise Physiology, Kent State University, 350 Midway Drive, 162 Macc Annex, Kent, OH, USA, Tel (330) 672-2930, Fax 330-672-2250 Email cfennelI@kent.edu
\end{abstract}

Received: July 28, 2016 | Published: August 09, 2016

\section{Introduction}

According to the American College of Sports Medicine (ACSM), most adults in the United States do not participate in the minimum amount of physical activity of 30 minutes of exercise five days per week, recommended for health benefits..$^{1-3}$ Additionally, the number of individuals remaining sedentary during free time is becoming a public health concern. ${ }^{1-3}$ The combination of low physical activity and increasing sedentary behavior is a growing problem. The prevalence of overweight and obese adults in the U.S. has rapidly increased in the recent years. In a study performed in 2012, 68.5\% of Americans were overweight, $34.9 \%$ were obese, and $6.4 \%$ were considered extremely obese. ${ }^{4}$ Additionally, $31.1 \%$ of the world is physically inactive. ${ }^{5}$ These data suggests that the major issue that needs to be addressed which is motivating people to be active so that health benefits may be achieved. However, studies have demonstrated that among individuals who begin an exercise routine, it is likely that $50 \%$ will discontinue exercise within six months. ${ }^{6}$ It is suggested that these behavior changes may not be able to occur without the assistance of health and fitness professionals. ${ }^{7}$ The structure of supervised fitness classes within an exercise program may be effective at improving fitness and decreasing risk factors associated with sedentary behavior. In addition, a supervised program may provide the structure needed for the individuals to then continue performing self-motivated physical activity.
One method of motivating people to be more active is through the use of visual feedback in the form of activity watches or other accelerometers. These devices provide visual feedback to encourage the user to meet the minimum recommended physical activity. The ACSM recommends a baseline standard of 10,000 steps per day to achieve the classification of physically active. ${ }^{8}$ While participants in exercise programs may believe they are increasing their activity level, there is also a rise in the "active couch potato" phenomenon. ${ }^{9}$ This results in an increase in activity during the workout, but a decrease in activity or return to previous behaviors for the rest of the day. To alter this behavior, a watch with visual feedback can provide motivation to meet certain milestones and encourage activity throughout the day. The MOV band is a validated accelerometer that can be worn on the wrist as a watch. It measures physical activity in the form of moves and is able to convert those numbers into miles via algorithms. It was previously validated against the Acti-graph accelerometer to be as accurate and reliable at recording and reporting movement. ${ }^{10}$ Despite the motivation that these devices provide, there is still a need for supervised programs to help directly engage people in an exercise program to increase their physical activity.

Previous literature has demonstrated that both supervised exercise and unsupervised exercise programs have been able to increase physical fitness. ${ }^{11-13}$ However, there are mixed results of whether supervised or unsupervised exercise is more effective. Many researchers have found that supervised programs are more beneficial 
in improving cardiovascular and muscular fitness than unsupervised interventions. ${ }^{11,14-16}$ There is a need to motivate inactive adults to exercise to reduce the risk of developing diseases related to sedentary behavior and to improve overall health. One investigation demonstrated that a supervised program increased energy expenditure and enhanced retention to the program at 6,12 , and 18 months compared to the unsupervised control group. ${ }^{11}$ Even at the later months, there was still significant participation in the exercise program compared to those still exercising unsupervised. Even though there will be attrition in any program, this study supports the idea that a supervised program may be an important factor in providing long-term motivation and maintaining a lifestyle that incorporates physical activity and exercise. Another study evaluated the influence of direct supervision during resistance training exercise on strength performance and found direct supervision to increase load and magnitude of results to a greater degree than an unsupervised group performing a similar routine. ${ }^{14}$ Additionally, an experiment conducted with health club members found a supervised exercise program with a personal trainer to be more beneficial in increasing lean body mass, strength, and VO2max than in a group working without supervision. ${ }^{16}$ Even with the added support of peers, the health club members found the supervision of the personal trainer to be a key factor in progressing with their fitness. In a cardiac rehabilitation setting, it was demonstrated that individuals were not able to sustain an unsupervised workout on their own and suggested that supervised programs may be necessary to achieve compliance to the prescribed workouts. ${ }^{15}$

The present investigation recruited previously sedentary individuals for a five month exercise program. The variables measured were muscular endurance, physical activity, and resting heart rate as a measure of cardiovascular fitness. In addition, the participants reported through questionnaires how the supervised program increased their self-efficacy and motivation for exercise in an unsupervised period for exercise immediately following the intervention. Our hypotheses were that

A. A five month supervised exercise program would increase muscular endurance and physical activity while decreasing resting heart rate and,

B. The loss of gains made would occur after the five weeks of unsupervised training.

\section{Methods}

\section{Participants}

Sixteen faculty and staff members (13 female, 3 male; age $48.7 \pm 11.0$ years) of a university-based exercise program volunteered to participate in this longitudinal study. This program was sponsored by Human Resources and the Exercise Physiology department and opens to all faculty and staff members on campus. Participants in the program were recruited through flyers hung around campus, emails, and word of mouth. The investigation was split into five months of supervised exercise classes followed by five weeks of unsupervised exercise. Prior to enrolling in the program, all participants submitted a Physician's Consent, Health History Questionnaire, and Exercise Questionnaire to participate in the exercise classes. Those that were willing to participate in the research study portion also filled out an Informed Consent approved by the Kent State University Institutional Review Board.

\section{Testing variables and outline}

This study was designed to look at the effects of a supervised exercise program on muscular endurance, physical activity, and resting heart rate in a previously sedentary population along with the effects of unsupervised exercise on maintaining the improvements made during the supervised program. The program began with pretesting fitness parameters prior to beginning the exercise classes. This testing included the partial curl-up, which measures the maximal number of curl-ups performed in 60 seconds, and push-ups to failure. These tests of muscular endurance were based on ACSM guidelines for exercise testing. ${ }^{8}$ and evaluated prior to beginning exercise classes. Post-testing took place after five months of classes at the conclusion of the supervised exercise. The final testing session occurred after the unsupervised portion of the study. This five month duration was used to provide sufficient time to allow the benefits of exercise to occur and for physical activity to be monitored. The five weeks period of time were selected to provide a sufficient amount of time to measure the effects that five weeks of unsupervised exercise would have, and it also coincided with the academic calendar as well.

\section{Movement activity}

To quantify physical activity, each participant was given a MOV band accelerometer upon enrollment in the program. The MOV band is a three-plane accelerometer that measures movement in multiple planes to give an accurate estimate of each participant's physical activity and was previously validated against the Acti-graph accelerometer. ${ }^{10}$ Physical activity was recorded as moves as a built-in algorithm was used to convert the movement data from moves to miles, which is more widely understood. Using the data given by the MOV band, physical activity could be expressed in terms that are familiar to the general population. This also helped to encourage participants to stay active throughout the day even after the exercise classes were completed. All participants were instructed to wear the MOV band as much as possible throughout the day, not just during exercise. Those who participated in this investigation wore the bands consistently from the beginning of the program until the end of the unsupervised period. This data was uploaded via USB to an online network where the researchers were able to access the data for analysis. This data was then used to evaluate the activity level of each participant throughout the day during the supervised and unsupervised periods of this study.

\section{Protocol}

Heart rate was measured through palpation of the radial artery by a trained researcher during the pre-testing and two post-testing sessions. Participants were seated for a minimum of five minutes to achieve a rested state. Research staff then palpated the radial artery and counted the pulse for 60 seconds. The research staff for each testing session was kept consistent to decrease variability between researchers.

Curl-ups were performed based on ACSM guidelines for physical fitness assessments. ${ }^{8}$ Participants lay supine on an exercise mat with their knees bent to 90oand the soles of their feet flat on the ground. Their arms lay by their side with the hands relaxed and the middle finger touching a piece of tape that was placed $10 \mathrm{~cm}$ from the end and parallel to the edge of the exercise mat. Participants were instructed to use the abdominal muscles to curl up such that their middle finger reached the edge of the exercise mat, then to return to the resting position. This motion was completed as many times as possible in 60 seconds without pausing. Research staff counted repetitions for each participant.

Push-ups were performed according to ACSM guidelines. ${ }^{8}$ Males were instructed to complete the test from their toes while females were to perform the push-ups from their knees. All participants were instructed to maintain a straight line from the shoulders through the 
hips to either the knees or toes. Research staff was supervising the test and commented on the individual's incorrect form. If the participant was not able to make the instructed correction, the test was ended. Participants were to complete as many push-ups as possible without pausing. A completed push-up counted if the arms were bent to $90^{\circ}$ in the down position and extended fully in the starting position. Participants were instructed to keep a steady pace for the duration of the test. Any pause or break in form was mentioned to the participant by the research staff. If the corrections were not made immediately, the test was ended. Research staff counted the number of repetitions. These protocols were followed for all three testing sessions, pretesting, post-testing, and follow-up testing. After completing the pretesting session, participants began the five month supervised exercise program.

\section{Exercise intervention}

The exercise intervention consisted of 60 minute classes three times per week for five months. The classes included a 10 minute warmup and five minute cool-down. Each day, participants could choose one of five class options to attend: jogging, boot camp, cardio dance, weight training, or low intensity circuits. All classes were led by one or two research staff that was trained in the specific area of exercise. Additional research assistants were present during the exercise sessions to offer input to the participants and ensure all participants were exercising safely. At the end of the supervised exercise program, the participants were instructed to continue independent exercise for the subsequent five weeks. The research staff provided examples of workouts and exercises to for the participants to complete during this time.

Three questionnaires were distributed throughout the course of the program. Upon enrollment in the program, the participants were given a survey asking about prior exercise participation and experience. At the end of the supervised exercise program before the transition to the unsupervised exercise portion, another questionnaire was distributed. This asked each participant to rate their self-efficacy for exercise after five months of supervised exercise classes and their willingness and intention to continue to be active on their own. The last questionnaire was distributed when the participants returned for follow-up testing after five weeks of independent exercise. This questionnaire asked each participant to report their compliance to exercising independently and whether they exceeded, met, or fell short of their anticipated exercise goals for the unsupervised period.

\section{Statistical Analysis}

The muscular endurance and physical activity data was entered into SPSS and analyzed using repeated measures ANOVA with three time points, pre-testing, post-testing, and the follow-up testing. The questionnaires were used as non-empirical data for participant anecdotal feedback and to evaluate reported compliance to exercise during the unsupervised part of the study.

\section{Results}

\section{Descriptive data}

Table 1 depicts the data for the participants at the three time points measured: pre-testing, post-testing, and after the five weeks unsupervised. The data was analyzed through repeated measures ANOVA and any significance was further analyzed through paired samples t-tests. Table 2 provides the data from pre supervised, post supervised, and unsupervised.
Table I Values are listed as means \pm standard deviation. There were thirteen female participants and three male participants.

\begin{tabular}{|lll|}
\hline Age (yrs) & Height (cm) & Pre-Test Weight (lbs) \\
\hline $48.7 \pm 11.0$ & $166.6 \pm 5.0$ & $201.0 \pm 48.6$ \\
\hline
\end{tabular}

Table 2 Pre Supervised, post Supervised, and Unsupervised data for push-ups, curl-ups, physical activity, and resting heart rate. Values are listed as means \pm standard deviation.

\begin{tabular}{|c|c|c|c|c|}
\hline & & $\begin{array}{l}\text { Supervised } \\
\text { Week I- } \\
\text { Pre Test }\end{array}$ & $\begin{array}{l}\text { Supervised } \\
\text { Week } 22\end{array}$ & $\begin{array}{l}\text { Unsupervised } \\
\text { Week } 27\end{array}$ \\
\hline Muscular & Push-ups (\#) & $19.0 \pm 12.1$ & $31.9 \pm 13.6$ & $23.2+14.3$ \\
\hline Endurance & Curl-ups (\#) & $33.8 \pm 9.7$ & $49.3 \pm 15.2$ & $42.8+19.2$ \\
\hline $\begin{array}{l}\text { Physical Activity } \\
\text { Behavior }\end{array}$ & $\begin{array}{l}\text { Physical Activity } \\
\text { (miles) }\end{array}$ & $\begin{array}{l}\text { No pre-test } \\
\text { data }\end{array}$ & $206.5 \pm 82.6$ & $150.5 \pm 65.5$ \\
\hline $\begin{array}{l}\text { Cardiovascular } \\
\text { Parameter }\end{array}$ & $\begin{array}{l}\text { Resting heart } \\
\text { rate (bpm) }\end{array}$ & $76.4 \pm 8.7$ & $72.4 \pm 9.1$ & $81.6 \pm 11.4$ \\
\hline
\end{tabular}

\section{Physical activity}

A repeated measures ANOVA indicated a main effect for miles $(\mathrm{F}=8.735, \mathrm{p}<0.001)$. Paired samples t-tests were used to determine where the main effect occurred. Figure 1 depicts the supervised

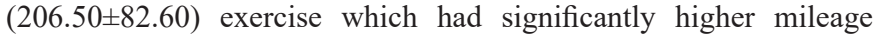
$(\mathrm{p}=0.003)$ than the unsupervised portion $(150.45 \pm 65.52)$.

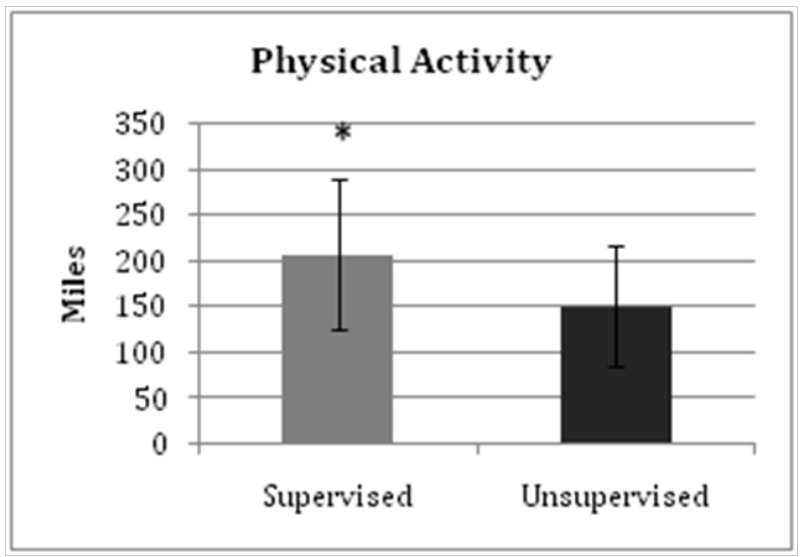

Figure I The amount of miles during the supervised and unsupervised portion of 5 weeks.

*Indicates significance between supervised and unsupervised sessions $(p=0.003)$.

\section{Muscular endurance}

For the muscular endurance changes, repeated measures ANOVA identified any main effects. There was a main effect for both pushups $(\mathrm{F}=7.573, \mathrm{p}=0.002)$ and curl-ups $(\mathrm{F}=8.541, \mathrm{p}<0.001)$. Figure 2 illustrates the push-ups using a paired samples t-test to indicate a significant difference $(p<0.001)$ between the number of push-ups performed prior to beginning supervised exercise $(19.00 \pm 12.12)$ and at the end of the supervised program (31.94 \pm 13.56$)$. There was no other significance for the push-up data. The paired samples t-tests for the curl-ups, illustrated in Figure 3, also indicated a significant difference $(\mathrm{p}<0.001)$ in the number performed at the end of the supervised exercise program $(49.31 \pm 15.15)$ compared to the beginning (33.75 \pm 9.74$)$

\section{Resting heart rate}

The repeated measures ANOVA and paired samples t-test (Figure $4)$ indicated there was a significant increase $(\mathrm{p}=0.005)$ in resting heart 
rate from the end of the supervised exercise program $(72.4 \pm 9.11 \mathrm{bpm})$ to the end of the unsupervised portion $(81.6 \pm 11.4 \mathrm{bpm})$. Resting heart rate was not significantly different after the unsupervised exercise than prior to beginning the supervised exercise program $(76.4 \pm 8.72 \mathrm{bpm})$.

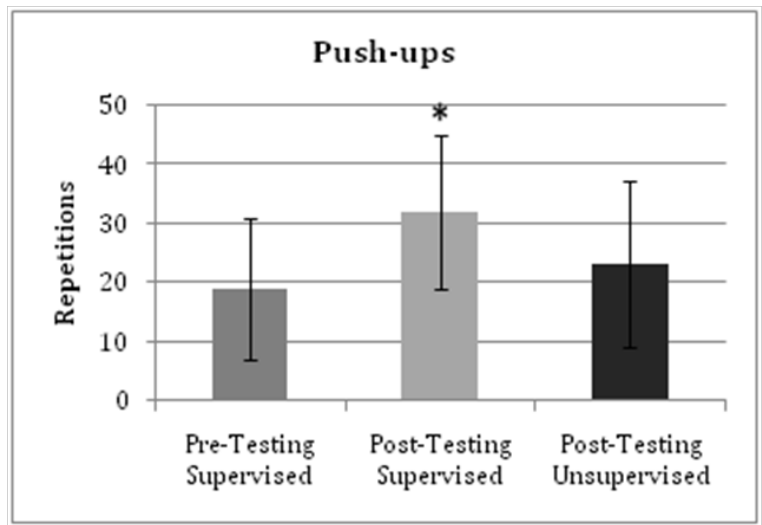

Figure 2 The amount of push-ups performed pre supervised; post supervised, and post unsupervised portions of the program.

*Indicates significance pre supervised to post supervised $(p<0.00 \mathrm{I})$.

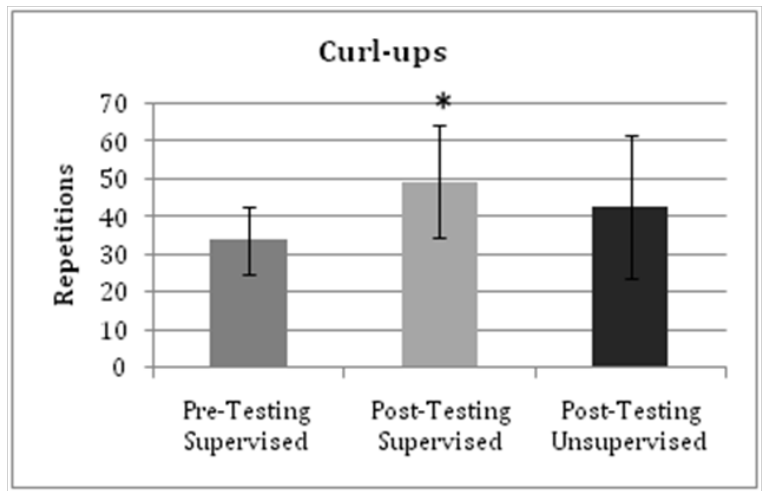

Figure 3 The number of curl-ups performed pre supervised; post supervised, and post unsupervised portions of the program.

*Indicates significance pre supervised to post supervised $(p<0.00 \mathrm{I})$.

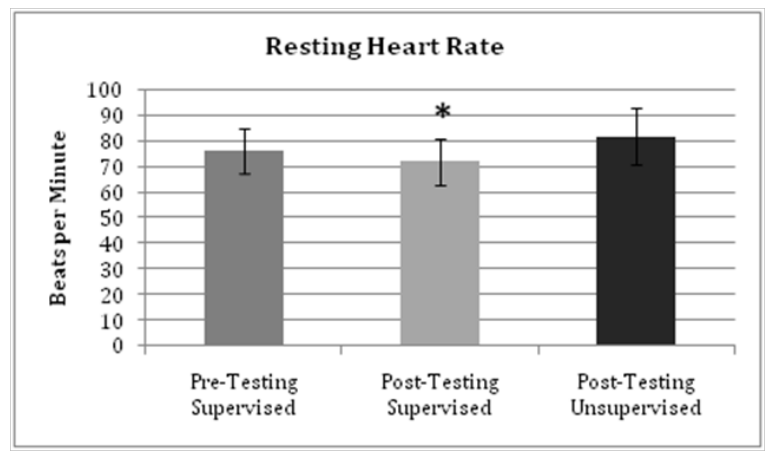

Figure 4 Resting heart rate pre, post supervised, and post unsupervised sessions of the program.

*Indicates significance post supervised to post unsupervised $(p=0.005)$.

\section{Discussion}

The purpose of this investigation was to evaluate the effectiveness of a supervised versus non-supervised exercise program on physical activity and muscular endurance as well as cardiovascular adaptation. This data demonstrated that a greater amount of physical activity was achieved during the supervised program compared to the unsupervised program. Additionally, during the supervised portion there was an increase in push-ups and curl-ups, but no difference in push-ups or curl-ups after the 5 weeks unsupervised compared to before the supervised portion. There was no change in heart rate after the 5 months of supervised exercise, but after the 5 weeks of unsupervised, there was a significant increase in heart rate. According to ACSM standards, the recommended amount of physical activity is 10,000 steps per day. This can be translated through validated algorithms to 5 miles of physical activity each day. The data from the MOV band showing the physical activity levels for each participant during the supervised exercise program indicates that the participants were averaging 5.8 miles each day. This is compared to the unsupervised portion of the study where activity levels dropped significantly to 4.3 miles. When the participants were instructed to exercise without the structured program, the average daily mileage decreased significantly. This is likely a reflection of the decreased exercise as well as total activity for the participants during the unsupervised portion of the study.

The muscular endurance data indicates that a supervised exercise program is more effective at improving this parameter compared to unsupervised exercise, which supports previous research. ${ }^{11,14-17}$ This muscular endurance data supports the effectiveness of a supervised exercise program compared to unsupervised exercise. Pre-testing data prior to beginning the supervised exercise program provided a baseline indication of both push-ups and curl-ups for the participants. Over the course of the supervised exercise program, participants increased both the number of push-ups and curl-ups that were able to be completed during post-testing. The initial number of pushups ranked the participants as a group in the 45th percentile or the Fair category based on ACSM standards. After participation in the exercise program, the participants ranked in the 80th percentile and were in the excellent category. A similar trend was observed for the curl-ups. The participants were in the Fair category prior to beginning the exercise program. During post-testing, after participation in the exercise program, the participants were ranked in the excellent category for the number of curl-ups performed in 60 seconds. This data supports the effectiveness of a supervised exercise program on increasing muscular endurance for both push-ups and curl-ups. After the unsupervised exercise period, the number of push-ups and curlups that the participants were able to complete was not significantly different from the number completed prior to beginning the supervised program. This indicates that participants may have decreased muscular endurance training during the unsupervised portion since the measured variables returned to baseline values after the period of unsupervised exercise.

Overall, the decrease in physical activity behavior and return of muscular endurance gains back to baseline may indicate that participants were not consistent with exercise during the unsupervised portion. Although not specifically measured, this may be caused from a lack of a behavior change to exercise independently after five months training, which supports previous data. ${ }^{17-19}$ Prior to beginning this exercise program, all participants were living selfdeclared sedentary lifestyles. The purpose of the program was to initiate a behavior change over the five months of supervised exercise that would carry into the five weeks without supervision. While this did not occur, all 16 participants continued to attend the supervised portion of exercise for five months, and then returned after the five weeks of non-supervision. This indicates that some form of a behavior change may have been made during the supervised exercise. Hence, these individuals may be motivated to participate in group, rather than independent exercise. 
The last variable measured for this study was resting heart rate. This was used as an indication of cardiovascular adaptations that occurred from consistent participation in exercise..$^{20}$ Although not significant, the resting heart rate at the end of the supervised exercise program was $3 \%$ lower than prior to beginning the program. There was a significant increase in resting heart rate from the end of the supervised exercise program to the end of the unsupervised portion of the study. Since heart rate did begin to lower over five months of training, this may indicate that the participants improved cardiovascular function, or parasympathetic tone, through participation in the exercise program, which supports previous research. ${ }^{1,12,20}$ The unsupervised portion resulted in those adaptations being lost and the participants reverting back to baseline cardiovascular parameters. This suggests the importance of a supervised exercise program on attaining beneficial cardiovascular changes; and the lack of and decrease of beneficial cardiovascular changes that may occur during unsupervised exercise. The small sample size may have contributed to the lack of statistical significance, and future studies with larger participation and longer duration may see significance in heart rate changes in response to exercise programs.

With obesity rates rising and while an estimated $68 \%$ of individuals do not participate in the minimal amount of physical activity recommended. ${ }^{1,12}$ there is a need for physical activity interventions to determine motivation strategies for individuals to exercise and increase movement. There are many ways to encourage exercise, however, a major question is how to establish a behavior change of long-term exercise adherence and whether just stressing the importance of movement will increase physical activity levels. Individuals must consider age, appreciate good health, have high self-efficacy, and believe in the importance of physical activity for long-term exercise adherence.$^{21}$ This study supports previous research on the benefits of a supervised exercise program. The MOV band data indicates that the participants were meeting ACSM recommendations for physical activity during the supervised portion. Muscular endurance, measured by assessing curl-ups and push-ups also improved based on ACSM standards. Potential cardiovascular adaptations may have begun changing throughout the program, although these changes were not sustained during the unsupervised period.

The second portion of the study was evaluating if participation in exercise program for the duration of five months would enable participants to be able to exercise independently, without the support of the group. This was not supported as there was a significant decrease in physical activity that was measured by the MOV band during the unsupervised portion. These results support previous data that five months is not a long enough duration to make a behavior change when participating in exercise while unsupervised. ${ }^{11}$ however it may be long enough to make a behavior change with supervised exercise, as participants continued the supervised program after the five week unsupervised portion. Testing that occurred after the unsupervised portion of the study indicated a return of muscular endurance levels to baseline as well as the loss of cardiovascular adaptations. This data suggests that not only is a supervised exercise program beneficial in motivating individuals to become more active and improve overall fitness, but that unsupervised exercise with only suggestions for activity and no oversight is not sufficient to result in similar improvements in fitness or even to maintain fitness. In addition, based on the questionnaires distributed to the participants at the end of the supervised exercise program and after the unsupervised period, the participants felt that they were not able to sustain regular exercise without the structure of the program. One participant specifically stated, "I need a group like this at all times." Another wrote, pertaining to the unsupervised portion, "I had planned to exercise 3-4 times a week, but only did 1-2 times. It was hard to keep motivated." One hundred percent of the participants stated they had a higher selfefficacy for exercise at the end of the supervised program. However, after the unsupervised period, only $24 \%$ of the participants felt as though they were able to sustain exercise independently. The other $76 \%$ stated that they either did not exercise at all or only exercised a few times and wished they had been able to do more. While this is qualitative evidence, it is still a major component in being able to motivate individuals to become more active and whether they are capable of following workouts without the structure of a supervised program.

A limitation of this research is the lack of a control group. While this exercise intervention occurred for all participants in the study, their previous sedentary behavior can act as a pseudo-control. Without the structure of the exercise program, nearly all the participants were self-reported sedentary and unable to sustain a regular workout routine. After enrolling in the program, the participants were able to increase physical activity levels to recommended levels. Future research is warranted with supervised exercise programs to determine what personality and motivational traits are associated with physical activity behavior. The use of objectively measured physical activity may be a more valid measurement of physical activity than selfreported data due to the subjective nature of the self reports.

\section{Conclusion}

This investigation suggests that supervised exercise programs in previously sedentary individuals are effective in improving overall fitness. In contrast, even after a period of five months of supervised exercise, in these individuals they were not equipped to exercise in an unsupervised setting and will not be able to maintain or improve their fitness without the support of a program. However, while the participants were unable to meet the recommendations of minimal exercise on their own without the structure of the program, they did demonstrate behavior to continue to perform exercise by enrolling in the next session of the program. This indicates a positive move in a direction that may lead to a continued increase in self-efficacy for exercise, as this structured exercise program was beneficial at improving physical activity behavior and health-related variables.

\section{Acknowledgments}

None.

\section{Conflicts of interest}

None.

\section{References}

1. Centers for Disease Control and Prevention Adult participation in aerobic and muscle-strengthening physical activities - United States, 2011. MMWR Morb Mortal Wkly Rep. 2013:62(17):326-330.

2. Centers for Disease Control and Prevention Adult participation in recommended levels of physical activity - United States, 2001 and 2003. MMWR Morb Mortal Wkly Rep. 2005:54(47):1208-1212.

3. Macera CA, Ham SA, Yore MM et al. Prevalence of Physical Activity in the United States: Behavior Risk Factor Surveillance System. Prev Chronic Dis. 2005:2(2):A1-A17.

4. Ogden CL, Carroll MD, Kit BK, Flegal KM et al. Prevalence of Childhood and Adult Obesity in the United States. JAMA. 2004:311(8):806-814.

5. Hallal PC, Andersen LB, Bull FC et al. Global physical activity levels: surveillance progress, pitfalls, and prospects. Lancet. 2012:380(9838):247-257. 
6. Dishman, RK, Morgan WP, O'Connor PJ Exercise Adherence: its impact on public health. Human Kinetics Books, Champaign, USA, pp. 1988:1-447.

7. Kahn EB, Ramsey LT, Brownson RC et al. The effectiveness of interventions to increase physical activity: a systematic review. $\mathrm{Am} J$ Prev Med. 2002:4(1):73-107.

8. Brad Ferguson American College of Sports Medicine Guidelines for Exercise Testing. ACSM's Guidelines for Exercise Testing and Prescription, (9th edn), Lippincott WIlliams and Wilkins, Philadelphia, USA, pp. 2014:1-328.

9. Owen N, Healy GN, Matthews CE et al.Too Much Sitting PopulationHealth Science of Sedentary Behavior. Exerc Sport Sci Rev. 2010:38(3):105-113.

10. Barkley JE, Rebold M, Carnes A et al. The validity of a commerciallyavailable, low-cost, wrist-mounted accelerometer during treadmill exercise. Med and Sci in Sport and Ex. 2013:46(5):490.

11. Cox KL, Burke V, Gorely TJ et al. Controlled Comparison of Retention and Adherence in Home vs Center Initiated Exercise Interventions in Women Ages 40-65 Years: The SWEAT Study (Sedentary Women Exercise Aderence Trial). Prev Med. 2003:36(1):17-29.

12. Perri MG, Martin AD, Leermakers EA Effects of group vs home based exercise in the treatment of obesity. J Consult Clin Psychol. 1997:65(2):278-285.

13. Stefanov T, Vekova A, Bonova I eta la. Effects of supervised vs nonsupervised combined aerobic and resistance exercise programme on cardiometabolic risk factors. Cent Eur J Public Health. 2013:21(1):8-16.
14. Mazzetti SA, Kraemer WJ, Volek JS et al. The Influence of Direct Supervision of Resistance Training on Strength Performance. Med Sci Sports Exerc. 2000:32(6):1175-1184

15. Nalini M, Moradi B, Esmaeilzadeh M et al. Does the Effect of Supervised Cardiac Rehabilitation Programs on Body Fat Distribution Remained Long Time. J Cardiovasc Thorac Res. 2013:5(4):133-138.

16. Storer TW, Dolezal BA, Berenc MN et al.Effect of Supervised, Periodized Exercise Training vs Self-Directed Training on Lean Body Mass and Other Fitness Variables in Health Club Members. J Strength Cond Res. 2014:28(7):1995-2006.

17. Yuza N, Ishida K, Miyamura M Cross transfer effects of muscular endurance during training and detraining. J Sports Med Phys Fitness. 2000:40(2):110-117.

18. Häkkinen K, Alén M, Komi PV Changes in isometric force and relaxation time, electromyographic and muscle fiber characteristics of human skeletal muscle during strength training and detraining. Acta Physiol Scand. 1985:125(4):573-585.

19. Mujika I, Padilla S Muscular Characteristics of Detraining in Humans. Med Sci Sports Exerc. 2001:33(8):1297-1303.

20. Kesaniemi YK, Danforth E, Jensen MD, et al. Dose-response issues concerning physical activity and health: an evidence-based symposium. Med Sci Sports Exerc. 2001:33(6):351-358.

21. McAuley E, Lox C, Duncan TE Long term maintenance of exercise, self-efficacy, and physiological change in older adults. J Gerontol. 1993:48(4):218-224. 